\title{
Role of the Wnt/ $\beta$-catenin signaling pathway in inducing apoptosis and renal fibrosis in 5/6-nephrectomized rats
}

\author{
XIN LIN $^{1 *}$, YAN ZHA ${ }^{1 *}$, XIANG-ZHEN ZENG ${ }^{2}$, RONG DONG ${ }^{1}$, QING-HUA WANG ${ }^{2}$ and DONG-TAO WANG ${ }^{3,4}$ \\ ${ }^{1}$ Department of Nephrology, People's Hospital of Guizhou Province, Guiyang, Guizhou 550002; ${ }^{2}$ Department of Nephrology, \\ Loudi Central Hospital, Loudi, Hunan 417000; ${ }^{3}$ Department of Nephrology, Shenzhen Traditional Chinese Medicine Hospital, \\ Guangzhou University of Traditional Chinese Medicine, Shenzhen, Guangdong 518033; 'Department of Nephrology, \\ Ruikang Affiliated Hospital, Guangxi University of Chinese Medicine, Nanning, Guangxi 530011, P.R. China
}

Received February 25, 2016; Accepted February 14, 2017

DOI: $10.3892 / \mathrm{mmr} .2017 .6461$

\begin{abstract}
Renal fibrosis is the final common pathway of all progressive renal disease. Excessive and chronic activation of the Wnt/ $\beta$-catenin signaling pathway results in chronic kidney disease (CKD) progression. To mimic CKD, the present study used 5/6-nephrectomized rats, and alterations in kidney histology, expression of $\beta$-catenin and renal cell apoptosis were assessed. In addition, mesangial cells were cultured in vitro and transfected with $\beta$-catenin siRNA to evaluate the effect of blocking Wnt/ $\beta$-catenin signaling on cell apoptosis and the expression of markers of renal fibrosis. The results demonstrated that CKD rat kidney tissues exhibited moderate renal fibrosis and significantly increased expression levels of $\beta$-catenin and apoptosis associated proteins compared with sham-operated rats. In vitro, silencing of $\beta$-catenin by siRNA attenuated tumor necrosis factor- $\alpha$-induced apoptosis and decreased mRNA expression levels of various markers of fibrosis, including fibronectin, transforming growth factor- $\beta$, and collagen I, III and IV. In conclusion, inhibition of $\mathrm{Wnt} / \beta$-catenin signaling by $\beta$-catenin silencing attenuated apoptosis and expression of fibrosis-associated markers in renal cells. The present study suggested that the $\mathrm{Wnt} / \beta$-catenin signaling pathway may serve as a potential treatment strategy for renal fibrotic disorders.
\end{abstract}

\section{Introduction}

Chronic kidney disease (CKD), defined as a progressive decline in renal function, has gradually become an important

Correspondence to: Dr Dong-Tao Wang, Department of Nephrology, Shenzhen Traditional Chinese Medicine Hospital, Guangzhou University of Traditional Chinese Medicine, 1 Fuhua Road, Shenzhen, Guangdong 518033, P.R. China

E-mail: wangdongtaoszzyy@163.com

*Contributed equally

Key words: chronic kidney disease, Wnt/ $\beta$-catenin signaling, apoptosis, fibrosis, renal problem of global public health during the past decades. It occurs in developed and developing countries. There are currently $>1.4$ million patients with CKD worldwide receiving renal replacement therapy (1).

Renal fibrosis is the final common pathway of all progressive renal disease. Various pathogenic factors stimulate the cells of the kidney to continually produce inflammatory mediators and cytokines, leading to chronic endothelial-mesenchymal transition (EMT) and extracellular matrix (ECM) production that eventually alters the normal structure and function of the nephron (2). The $\mathrm{Wnt} / \beta$-catenin signaling pathway is important for the regulation of cell adhesion, migration, EMT, embryonic development and homeostasis of tissues and organs. Dysregulation of this important signaling pathway is closely associated with the progression of renal fibrosis (3). Transforming growth factor (TGF)- $\beta$ is typically regarded as the most potent profibrogenic cytokine and a central mediator of renal fibrosis (4). TGF- $\beta$ is a primary ECM regulator, acting as an inducer of ECM synthesis and an inhibitor of ECM degradation, via the direct suppression of matrix metalloproteinases and induction of tissue inhibitors of metalloproteinases (5). In addition, TGF- $\beta$ may induce tubular EMT and directly contribute to the myofibroblast pool responsible for interstitial matrix production (6).

Previous studies have demonstrated various points of cross-talk between the Wnt/ $\beta$-catenin signaling pathway and TGF- $\beta$ in the development of tissue fibrosis and tumor progression. Wnt $/ \beta$-catenin signaling affects the TGF- $\beta$ signaling pathway in a multi-level synergistic manner in various settings of fibrosis, and TGF- $\beta$ signaling induces activation of the Wnt/ $\beta$-catenin signaling pathway, as the two pathways regulate ligand generation of the other pathway $(7,8)$.

Activated $\mathrm{Wnt} / \beta$-catenin signaling has been demonstrated to promote fibroblast activities in various organs, including the kidneys, which indicates that this pathway may be reactivated following tissue injury (9-12). Strategies targeting the Wnt/ $\beta$-catenin signaling pathway by Wnt modulation in various animal models of fibrosis have proven to be promising. Using a peptidomimetic small molecule, ICG-001, which disrupts CREB-binding protein-catenin-mediated gene transcription, suppressed expression of fibrosis-associated proteins and ameliorated renal interstitial fibrotic injury in kidneys (13). 
However, whether inhibiting the Wnt/ $\beta$-catenin signaling pathway reverses fibrosis remains controversial. Previous studies have provided evidence that inhibition of $\mathrm{Wnt} / \beta$-catenin signaling may ameliorate fibrosis and injury (14-16). However, a separate study revealed that activation of the $\beta$-catenin signaling pathway in alveolar epithelial type 2 (AT2) cells contributes to epithelial repair following injury, and depletion of $\beta$-catenin by Cre recombinase promotes fibrosis (17).

Therefore, the aim of the present study was to evaluate the role of Wnt/ $\beta$-catenin signaling on kidney cell apoptosis and the expression of markers of renal fibrosis.

\section{Materials and methods}

Animals and experimental design. All experimental protocols were approved by the ethics committee of The People's Hospital of Guizhou Province (Guiyang, China), and followed the guidelines of Care and Use of Laboratory Animals formulated by the Ministry of Science and Technology of China. Male Sprague-Dawley rats [n=20; weight, 180-220 g; certification number, SCXK (Yue) 2006-0015] were purchased from the Experimental Animal Center of Southern Medical University (Guangzhou, China) and were randomly divided into sham and CKD groups ( $n=10$ per group), and given free access to food and water in a room with constant temperature $\left(20 \pm 1^{\circ} \mathrm{C}\right)$ and a $12 \mathrm{~h}$ light/dark cycle. Using full sterile technique, all rats underwent sham surgery or $5 / 6$ nephrectomy under anesthesia with 3\% isoflurane (RWD Life Science Co., Ltd, Shenzhen, China) in $\mathrm{N}_{2} \mathrm{O} / \mathrm{O}^{2}(2: 1)$, as previously described (18). Briefly, the upper and lower poles of the left kidney were removed using a retroperitoneal approach and hemostatic absorbable collagen sponges were used to stop the bleeding. The right kidney was removed one week later. In the sham-operated rats, laparotomy was performed without 5/6 nephrectomy. All rats survived to the experimental endpoint of 12 weeks after the operation. Rats were anesthetized by administration of $3 \%$ isoflurane in $\mathrm{N}_{2} / \mathrm{O}^{2}(2: 1)$ prior to sacrifice. Immediately after the rats were sacrificed, the kidneys were dissected and rinsed in phosphate buffered saline. The $10 \%$ formalin-fixed kidney and frozen sections were subsequently used for histopathological examination and immunohistochemical studies.

Culture of mesangial cells. The rat mesangial cell line HBZY-1 was obtained from the Wuhan University Cell Bank (Wuhan, China), and cultured in Dulbecco's modified Eagle's medium (PromoCell GmbH, Heidelberg, Germany) supplemented with $5 \mathrm{mM}$ glucose and $10 \%$ heat-inactivated fetal calf serum (Sigma-Aldrich; Merck KGaA, Darmstadt, Germany), at $37^{\circ} \mathrm{C}$ in $5 \% \mathrm{CO}_{2}$.

Histology and immunofluorescence. All samples were fixed in $4 \%$ buffered paraformaldehyde, decalcified in $50 \mathrm{mM}$ EDTA, embedded in paraffin and sectioned at a thickness of $5 \mathrm{~mm}$. The sections were processed for periodic acid-Schiff (PAS), hematoxylin and eosin (H\&E), periodic acid silver methenamine (PASM), Masson's trichrome and immunofluorescence staining.

ForH\&E staining, sections were incubated with hematoxylin (Mayer's hematoxylin solution; VWR International, Radnor,
PA, USA) for $15 \mathrm{~min}$, washed in water for $5 \mathrm{~min}$, and stained with eosin (VWR International) for $1 \mathrm{~min}$.

For PAS Staining, sections were fixed in $95 \%$ alcohol for 10 min prior to being washed, dried, stained with $1 \%$ periodic acid for $20 \mathrm{~min}$, and washed and dried again. Each section was subsequently stained with Schiff's reagent for $60 \mathrm{~min}$, washed and dried, stained with hematoxylin for $5 \mathrm{~min}$, and washed and dried.

For PASM staining, sectionswere immersed in $0.005 \mathrm{M}$ periodic acid for $20 \mathrm{~min}$ at $22^{\circ} \mathrm{C}$, rinsed twice in distilled water and stained for $70 \mathrm{~min}$ at $50^{\circ} \mathrm{C}$ in freshly-made $20 \mathrm{ml}$ silver-methenamine solution $(0.25 \%$ silver nitrate, $0.3 \mathrm{~g}$ hexamethylenetetramine, $8 \mathrm{ml} \mathrm{5 \%}$ sodium borate and $12 \mathrm{ml}$ distilled water). Following staining, sectionswere rinsed four times in distilled water, treated with $0.5 \%$ sodium thiosulfate for $2 \mathrm{~min}$ at $22^{\circ} \mathrm{C}$, washed twice in distilled water and dried on filter paper.

For Masson's trichrome staining, a Masson-Goldner Staining kit (Merck KGaA, Darmstadt, Germany) was used, according to the manufacturer's protocol. Nuclei were stained with hematoxylin for 15 min using Weigert's Iron Hematoxylin kit (VWR International), followed by azophloxin staining for 15 min. Samples were washed with acetic acid (1\%) and placed in acid Orange $\mathrm{G}$ solution. Samples were rinsed with $1 \%$ acetic acid for $30 \mathrm{sec}$, stained with Light Green for $5 \mathrm{~min}$ and rinsed again in $1 \%$ acetic acid for $5 \mathrm{~min}$.

For immunofluorescence staining, sections were blocked with 5\% normal sheep serum (Sigma-Aldrich; Merck $\mathrm{KGaA}$ ), in PBS for $45 \mathrm{~min}$ at room temperature and incubated with a monoclonal mouse anti-rat $\beta$-catenin antibody (RM-2101-RQ;1:200; Thermo Fisher Scientific, Inc., Waltham, MA, USA) at $4^{\circ} \mathrm{C}$ overnight. The reaction was visualized under a fluorescence microscope following incubation with a chicken anti-mouse Texas Red-conjugated IgG secondary antibody (sc-2954; 1:100; Santa Cruz Biotechnology, Inc., Dallas, TX, USA). A substitution of primary antibody with $10 \%$ non-immune goat serum (Invitrogen; Thermo Fisher Scientific, Inc.) was used as a negative control.

Terminal dUTP nick end labelling analysis. DNA strand breaks were assessed by fluorescent labeling of terminal dUTP nick end labeling (TUNEL), using the One Step TUNEL apoptosis assay kit (Beyotime Institute of Biotechnology, Haimen, China) as previously described (18). Images were captured with a Nikon DXM 1200C camera using Nikon ACT-1C software (Nikon Corporation, Tokyo, Japan). Within the delineated areas (measured and assisted with Stereo Investigator software v.10.5; MBF Bioscience, Williston, VT, USA), TUNEL-positive cells were counted, and their densities were calculated.

Reverse transcription-quantitative polymerase chain reaction (RT-qPCR). Total RNA was extracted from the renal cortex using TRIzol reagent (Invitrogen; Thermo Fisher Scientific, Inc.) and dissolved in diethylpyrocarbonate-treated water. Total RNA was reverse transcribed to cDNA using $50 \mathrm{ng}$ of total RNA in a $20 \mu \mathrm{l}$ reaction mixture using a RevertAid First Strand cDNA Synthesis kit (Thermo Fisher Scientific, Inc.), each reaction contained $200 \mathrm{U}$ RevertAidM-MuLV reverse transcriptase. Quantitative PCR was performed using 
the SYBR-Green method in atotal reaction mixture of $20 \mu 1$ containing 0.2 U of JumpStart Taq polymerase (Sigma-Aldrich; Merck KGaA), 10 pmol of each forward and reverse primer, and $1 \mu \mathrm{l}$ cDNA in a MyiQ real-time PCR detection system (Bio-Rad Laboratories, Inc., Hercules, CA, USA) in triplicate. The thermocycling conditions were as follows: Initial melting, $98^{\circ} \mathrm{C}$ for $30 \mathrm{sec} ; 35$ cycles of $98^{\circ} \mathrm{C}$ for $10 \mathrm{sec}, 62^{\circ} \mathrm{C}$ for $30 \mathrm{sec}$, $72^{\circ} \mathrm{C}$ for $2 \mathrm{~min}$; final extension, $72^{\circ} \mathrm{C}$ for $10 \mathrm{~min}$; hold, $4-10^{\circ} \mathrm{C}$. Sequences of specific primers were as follows: collagen I, forward, 5'-ATCCTGCCGATGTCGCTAT-3' and reverse, 5'-CCACAAGCGTGCTGTAGGT-3'; collagen III, forward, 5'-CTGGTCCTGTTGGTCCATCT-3' and reverse, 5'-ACC TTTGTCACCTCGTGGAC-3'; collagen IV, forward, 5'-GCC CTACGTTAGCAGATGTACC-3' and reverse, 5'-TATAAA TGGACTGGCTCGGAAT-3'; $\beta$-catenin, forward, 5'-ATT TGATGGAGTTGGACATGGC-3' and reverse, 5'-GAGGAA GAGGATGTGGATACCTCC-3'; fibronectin, forward 5'-GTG ATCTACGAGGGACAGC-3', and reverse 5'-GCTGGTGGT GAAGTCAAAG-3'; TGF- $\beta$, forward, 5'-GCCAGATCCTGT CCAAACTAA-3', and reverse, 5'-TTGTTGCGGTCCACC ATTA-3'. GAPDH, forward, 5'-ATGCTGGTGCTGAGTATG TC-3' and reverse, 5'-AGTTGTCATATTTCTCGTGG-3. Detected levels of target mRNAs were calculated using the $\triangle \Delta \mathrm{Cq}$ method (19) and normalized to GAPDH in arbitrary units.

Western blot analysis. Kidney tissue ( 40 mg) was snap-frozen and ground in a mortar, thawed and homogenized in lysis buffer (\#9803, Cell Signaling Technology, Inc., Danvers, MA, USA) supplemented with $8 \mu$ l protease inhibitor cocktail (Calbiochem; Merck KGaA) and phosphatase inhibitor cocktail (Sigma-Aldrich; Merck KGaA), and centrifuged at $15,000 \mathrm{x}$ g for $30 \mathrm{~min}$ at $4^{\circ} \mathrm{C}$. Protein concentrations were determined by a modified Bradford assay using a Bio-Rad microtiter plate (Bio-Rad Laboratories, Inc.). Equal amounts of protein $(50 \mu \mathrm{g})$ were loaded onto $10 \%$ sodium dodecyl sulfate-polyacrylamide gels for separation, transferred to polyvinylidene difluoride membranes and blocked at room temperature for $1 \mathrm{~h}$ in $5 \%$ non-fat milk in Tris-buffered saline (100 mM NaCl, $50 \mathrm{mM}$ Tris $\mathrm{pH} 7.5$ ) containing $0.1 \%$ Tween-20. Membraneswere incubated with primary antibodies targeting $\beta$-catenin (rabbit monoclonal antibody (mAb); \#8480; 1,1000), cleaved caspase-3 (rabbit mAb; \#9654; 1:1,000), B-cell lymphoma 2 (Bcl-2) -associated X protein (Bax) (rabbit mAb; \#14796; 1,1000) (all from Cell Signaling Technology, Inc.), Bcl-2 (rabbit mAb; sc-509; 1,200; Santa Cruz Biotechnology) and GAPDH (mousemAb; 60004-1-Ig, 1:1,000, ProteinTech Group, Inc., Chicago, IL, USA) overnight at $4^{\circ} \mathrm{C}$. Membranes were subsequently incubated with sheep anti-mouse immunoglobulin (Ig)-G(RPN4201; GE Healthcare Bio-Sciences, Pittsburgh, PA, USA) and goat anti-rabbit IgG (RPN4301, GE Healthcare Bio-Sciences) horseradish peroxidase-conjugated secondary antibodies, diluted 1:10,000, for $2 \mathrm{~h}$ at room temperature. Protein signals were detected using an Enhanced Chemiluminescence reagent (GE Healthcare Bio-Sciences) and images were captured using Image Station 2000MM (Kodak, Rochester, NY, USA). Densitometry of the blot images was performed using Molecular Imaging Software version 4.0 (Kodak).
Transfection. According to the sequence in the study of Verma et al (20), small interfering RNA (siRNA) targeting $\beta$-catenin (CTNNB1, NM001904) was synthesized by Invitrogen; Thermo Fisher Scientific, Inc. The sense was 5'-CCA UGC UGU AUA CUC CAC AGG AAA U-3', which extends between amino acids 79 and 85 of $\beta$-catenin. A scrambled siRNA without homology to any mammalian gene sequence served as a negative control. The sense was: 5'-GUCAUUGACUUAUCG AUGGdTdT-3'. Mesangial cells in the logarithmic phase were harvested by trypsinization and seeded at $1 \times 10^{6}$ cells/well into 6-well plates to yield $80-90 \%$ confluence. Transfections were performed using Lipofectamine ${ }^{\circledR} 2000$ (Invitrogen; Thermo Fisher Scientific, Inc.) with $100 \mathrm{nM} \beta$-catenin or scramble control siRNA (silencer FAM-labeled scramble negative control), according to the manufacturer's protocol. Transfected cells were incubated overnight under normal growth conditions, the medium was subsequently replaced and cells were stimulated with $2.5 \mathrm{ng} / \mathrm{ml}$ TNF- $\alpha$ (R\&D Systems, Inc., Minneapolis, MN, USA) or vehicle control (Sterile PBS containing $0.1 \%$ bovine serum albumin (Tocris Bioscience, Bristol, UK) ) for $24 \mathrm{~h}$. Morphological alterations indicating apoptosis were observed by Hoechst 33258 staining (Nanjing KeyGen Biotech Co., Ltd., Nanjing, China) under a fluorescence microscope, and quantified using Image J software v1.32 (National Institutes of Health, Bethesda, MD, USA) (21).

Statistical analysis. Data are presented as the mean \pm standard error. Statistical analysis was performed using SPSS software version 19.0 (IBM SPSS, Armonk, NY, USA). For data that were normally distributed, one-way analysis of variance (ANOVA) was performed followed by pair-wise comparison using the least-significant difference post hoc test. For data that were unequal and nonparametric, one-way ANOVA followed by Dunn's method for pair-wise comparison was used. $\mathrm{P}<0.05$ was considered to indicate a statistically significant difference.

\section{Results}

Histological and protein expression alterations in kidney tissues from CKD rats. As demonstrated in Fig. 1A, renal histology in sham-operated rats (control group) was normal. However, histological examination of kidney tissues from 5/6-nephrectomized rats (CKD group) revealed a moderate glomerular mesangial cell proliferation and glomerular ECM accumulation, localized or diffuse. Segmental sclerosis of capillary vessels, capsular synechia and tubular atrophy were additionally observed. Masson's trichrome staining demonstrated prominent interstitial fibrosis and extensive inflammatory cell invasion in the CKD group compared with the control group. The mRNA expression levels of markers of mesangial expansion and tubulointerstitial fibrosis were examined by RT-qPCR. TGF- $\beta$, fibronectin, and collagen I, III and IV mRNA expression levels were significantly upregulated, by 3.2-, 5.3-, 2.2-, 1.8- and 5.6-fold $(\mathrm{P}<0.05)$, respectively, in CKD rat kidney tissues compared with the control group (Fig. 1B).

Expression of $\beta$-catenin in kidney tissues from CKD rats. Expression of $\beta$-catenin was examined by immunofluorescence staining in kidney tissue sections from CKD and 

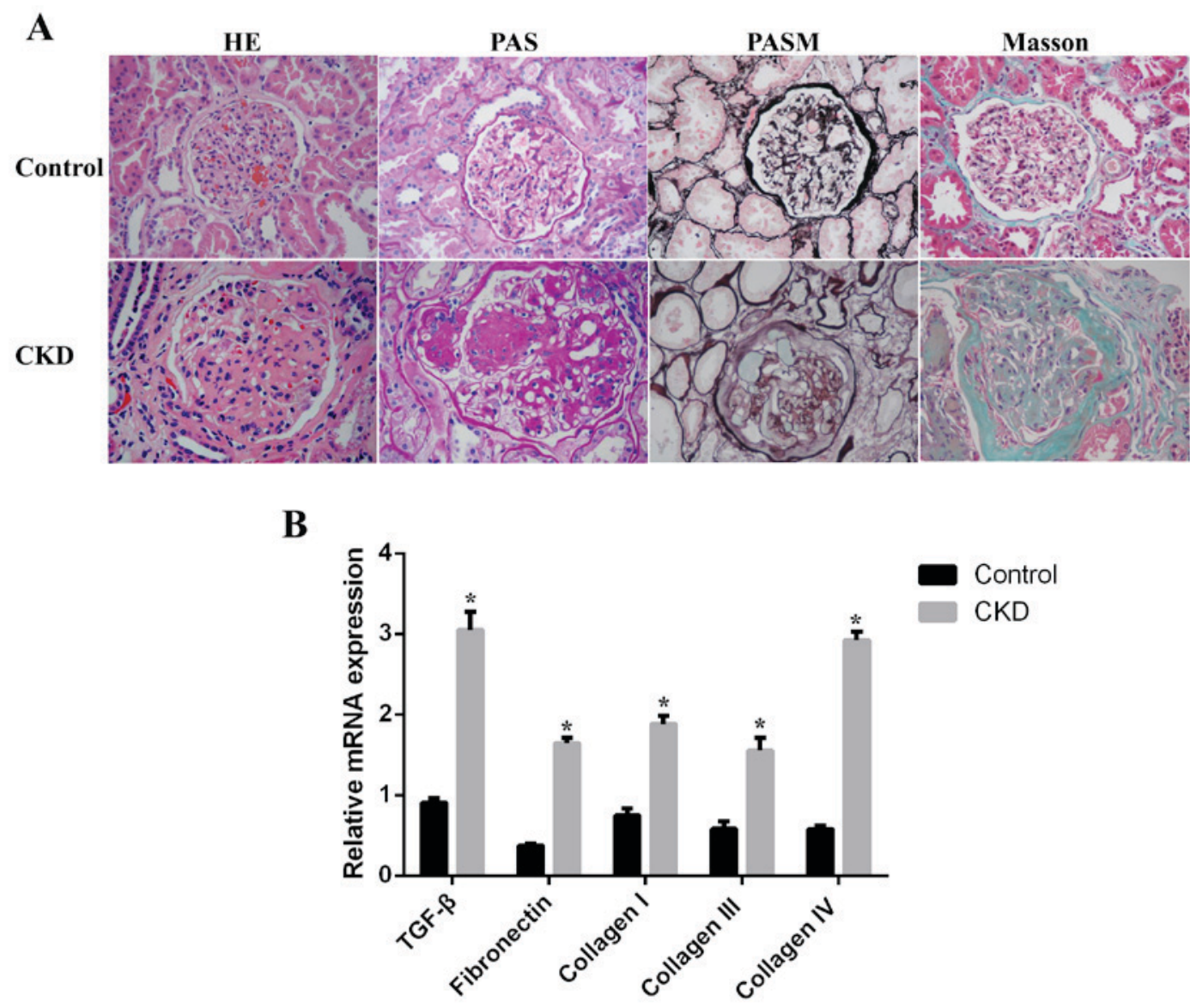

Figure 1. Histological assessment of kidneys from CKD rats. (A) Paraffin sections of kidney tissues from control (sham-operated) and CKD (5/6-nephrectomized) rats were stained with H\&E, PAS, PASM or Masson's trichrome. Original magnification, $x 400$. (B) mRNA expression levels of TGF- $\beta$, fibronectin, and collagen I, III and IV in control and CKD kidney tissues. Data are expressed as the mean \pm standard error ( $\mathrm{n}=8$ per group). " $\mathrm{P}<0.05$ vs. control. CKD, chronic kidney disease; H\&E, hematoxylin and eosin; PAS, periodic acid-Schiff; PASM, periodic acid silver methenamine; TGF- $\beta$, transforming growth factor- $\beta$.

control rats. $\beta$-catenin staining was barely observed in the kidney tissues of control rats, but was markedly increased in the kidneys of CKD rats (Fig. 2A). Western blotting revealed that protein expression levels of $\beta$-catenin were significantly increased by 2.8 -fold $(\mathrm{P}=0.002)$ in $\mathrm{CKD}$ group kidney tissues compared with the control group (Fig. 2B). Assessment of mRNA expression levels by RT-qPCR revealed a significant 3.1-fold $(\mathrm{P}<0.001)$ increase in $\beta$-catenin mRNA expression levels in the CKD compared with the control group (Fig. 2C).

Renal cell apoptosis in CKD rats. Renal cell apoptosis was examined in kidney tissues from CKD and control rats by terminal deoxynucleotidyl transferase UTP nick-end labeling (TUNEL) assay. As demonstrated in Fig. 3, although TUNEL staining was almost absent in kidney sections from the control group, it was present in those from CKD rats (Fig. 4A). The expression levels of the apoptosis-associated proteins cleaved caspase-3, Bax and Bcl-2 were examined by western blotting in kidney tissues from CKD and control rats. Protein expression levels of cleaved caspase-3 and Bax were significantly increased and levels of Bcl-2 were significantly decreased in CKD rat kidney tissues compared with the control group (Fig. 3B).

Silencing of $\beta$-catenin attenuates apoptosis. As CKD rat kidney tissues exhibited increased expression levels of $\beta$-catenin and apoptosis-associated proteins, the hypothesis that $\beta$-catenin has an active role in regulating apoptosis in renal cells was further tested. Expression of $\beta$-catenin was silenced by siRNA in mesangial cells in vitro. The mRNA expression levels of $\beta$-catenin in mesangial cells were significantly decreased following $\beta$-catenin siRNA transfection compared with scrambled control-transfected cells (Fig. 4A). Mesangial cells were subsequently stimulated with TNF- $\alpha$, an inducer of apoptosis, for $24 \mathrm{~h}$. Expression of $\beta$-catenin was successfully silenced by siRNA in the TNF- $\alpha$-stimulated cells compared with the scramble control-transfected cells (Fig. 4A). Assessment of apoptosis by Hoechst nuclear staining demonstrated that TNF- $\alpha$-treated cells exhibited concentrated cell nuclei and numerous dead cells with collapsed plasmalemma and solved karyotheca were observed, compared with vehicle-treated cells (Fig. 4B). Quantification of the staining revealed that the apoptosis rate was significantly greater in the TNF- $\alpha$ group compared with the vehicle group (Fig. 4C). However, $\beta$-catenin silencing significantly inhibited TNF- $\alpha$-induced apoptosis in mesangial cells, compared with scrambled control-transfected cells, suggesting that $\beta$-catenin may be important in regulating apoptosis. Western blotting revealed that the expression levels of the pro-apoptotic proteins cleaved caspase-3 (Fig. 4D) and Bax (Fig. 4E) were decreased in the $\beta$-catenin siRNA + TNF- $\alpha$ group compared with the scrambled control $+\mathrm{TNF}-\alpha$ group, 


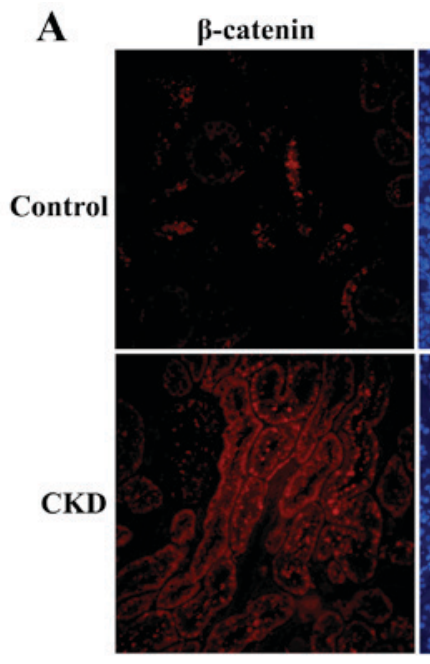

B
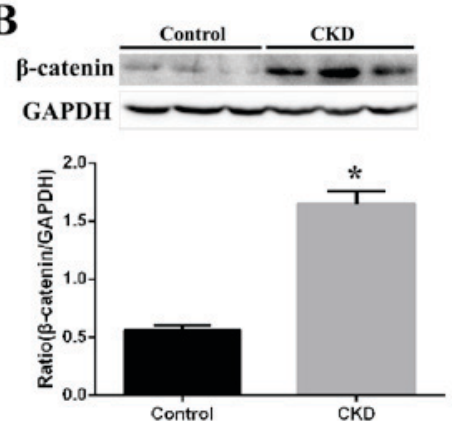

DAPI

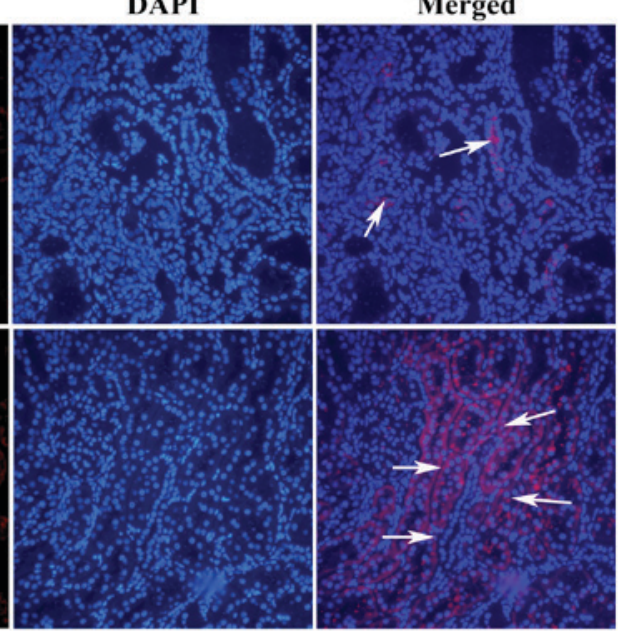

C

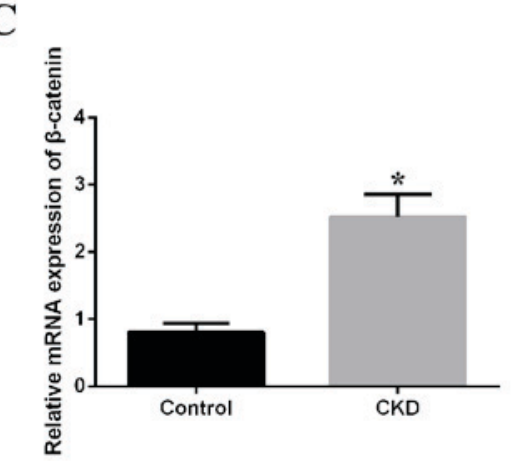

Figure 2. $\beta$-catenin expression in kidneys from CKD rats. (A) Immunofluorescence staining of $\beta$-catenin (red) in kidney tissue sections from control (sham-operated) and CKD (5/6-nephrectomized) rats. Nuclei were counterstained with DAPI (blue). $\beta$-catenin-positive areas are indicated by arrows. Original magnification, $\mathrm{x} 400$. (B) Representative western blot images and quantification of $\beta$-catenin protein expression levels in kidney tissues from control and CKD groups. (C) Quantification of reverse transcription-quantitative polymerase chain reaction results for $\beta$-catenin mRNA expression levels in kidney tissues from control and CKD groups. Data are expressed as the mean \pm standard error ( $\mathrm{n}=8$ per group). ${ }^{*} \mathrm{P}<0.05$ vs. control. $\mathrm{CKD}$, chronic kidney disease.

2.1 and 1.5-fold $(\mathrm{P}<0.05)$, respectively. By contrast, expression levels of the anti-apoptotic protein Bcl-2 were increased 1.6 -fold in the $\beta$-catenin siRNA+ TNF- $\alpha$ group compared with the scrambled control + TNF- $\alpha$ group (Fig. 4F).

Silencing of $\beta$-catenin alleviates fibrosis. The role of $\beta$-catenin in renal fibrosis was assessed by determining the effect of $\beta$-catenin silencing on TNF- $\alpha$-induced expression of fibrotic markers in mesangial cells in vitro. Treatment of mesangial cells with TNF- $\alpha$ significantly upregulated mRNA expression levels of fibronectin (Fig. 5A), TGF- $\beta$ (Fig. 5B), and collagen I (Fig. 5C), III (Fig. 5D) and IV (Fig. 5E) compared with vehicle-treated cells. Silencing of $\beta$-catenin by siRNA prior to TNF- $\alpha$ treatment, however, significantly decreased the mRNA expression levels of all fibrotic markers assessed. The mRNA expression levels of fibronectin, TGF- $\beta$, and collagen I, III and IV decreased by 2, 2.9,2.4, 2.1 and 3.1-fold $(\mathrm{P}<0.05)$, respectively, in the $\beta$-catenin siRNA + TNF- $\alpha$ group compared to the scrambled control $+\mathrm{TNF}-\alpha$ group.

\section{Discussion}

The evolutionarily conserved Wnt/ $\beta$-catenin signaling pathway is a complex and critical developmental pathway that has crucial roles in maintaining tissue homeostasis and initiating organ repair following injury (22). Previous studies have demonstrated a protective role for $\mathrm{Wnt} / \beta$-catenin signaling in healing and repair following acute kidney injury $(23,24)$; however, excessive and continuous $\mathrm{Wnt} / \beta$-catenin signaling may result in renal fibrosis and CKD progression. Chronic and progressive upregulation of $\beta$-catenin is a final pathway common to a wide variety of fibrotic CKDs $(25,26)$, including diabetic nephropathy, obstructive nephropathy, adriamycin nephropathy, chronic allograft nephropathy, polycystic kidney disease and, as demonstrated in the present study, remnant 5/6-nephrectomy. A recent study has indicated that sustained activation of $\mathrm{Wnt} / \beta$-catenin signaling induces renal interstitial fibroblast activation and promotes fibronectin expression, resulting in the progression from acute kidney injury to CKD in ischemia/reperfusion injury mice (27).

The present results indicated that expression of $\beta$-catenin, and the apoptosis-associated proteins cleaved caspase- 3 and Bax, were significantly increased in the kidneys of CKD rats compared with controls. By contrast, expression of the anti-apoptotic protein Bcl-2 was decreased in the kidneys of CKD rats compared with controls. This is consistent with a previous study, which demonstrated that the Wnt/ $\beta$-catenin signaling pathway is closely associated with mesangial cell apoptosis (28). The present study suggested that renal tissue from CKD rats exhibited increased Wnt/ $\beta$-catenin signaling and increased apoptosis compared with control rats.

Previous studies have revealed cross-talk between the Wnt/ $\beta$-catenin signaling pathway and other fibrotic pathways, 


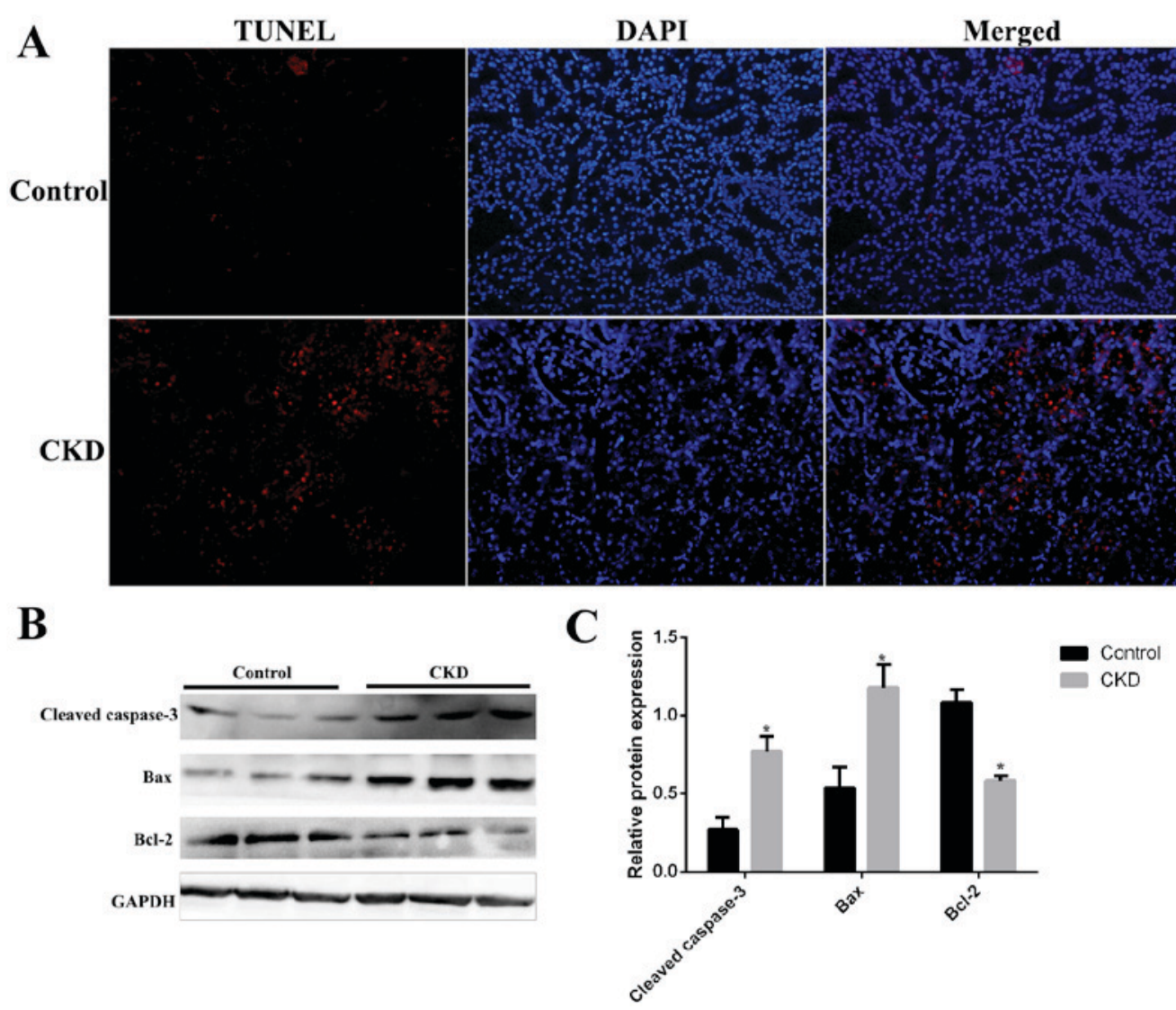

Figure 3. Apoptosis is increased in kidneys from CKD rats. (A) Representative images of TUNEL staining (red) in kidney sections from control (sham-operated) and CKD (5/6-nephrectomized) rats. Nuclei were counterstained with DAPI (blue). Original magnification, x400. (B) Representative western blot images and (C) quantification of cleaved caspase-3, Bax and Bcl-2 protein expression levels in kidney tissues from control and CKD groups. GAPDH served as a loading control. Data are expressed as the mean \pm standard error ( $\mathrm{n}=8$ per group). ${ }^{\mathrm{P}}<0.05$ vs. control. $\mathrm{CKD}$, chronic kidney disease; TUNEL, terminal deoxynucleotidyl transferase UTP nick-end labeling; Bax, B-cell lymphoma 2-associatedX protein; Bcl-2, B-cell lymphoma 2.

including TGF- $\beta$ signaling, and confirmed that certain differentially expressed microRNAs targeting these pathways lead to tissue injury and fibrosis $(29,30)$. These studies suggested that the $\mathrm{Wnt} / \beta$-catenin signaling pathway is important in renal fibrogenesis (3). In the tubulointerstitium and glomeruli, hyperactive $\mathrm{Wnt} / \beta$-catenin signaling exerts its effects via induction of target fibrosis-associated genes, including snail family transcriptional repressor 1 , fibronectin, plasminogen activator inhibitor-1, twist family bHLH transcription factor 1 and matrix metalloproteinase-7 (22). Of these fibrosis-associated genes, the role of fibronectin in fibrosis has long been established. In the present study, mRNA expression levels of fibronectin and various collagen genes were demonstrated to be significantly increased in kidney tissues from CKD rats compared with control rats.

Previous studies have confirmed that a variety of strategies to block Wnt $/ \beta$-catenin signaling, including at the cellular, cytoplasmic and nuclear levels, protect against renal fibrosis $(12,13,31)$. The present study demonstrated the role of the $\mathrm{Wnt} / \beta$-catenin signaling pathway in inducing apoptosis and renal fibrosis in 5/6-nephrectomized rats. Silencing of $\beta$-catenin in mesangial cells in vitro using siRNA decreased the mRNA expression levels of the pro-apoptotic proteins cleaved caspase- 3 and Bax, and of the fibrosis-associated proteins fibronectin, TGF- $\beta$, and collagen I, III and IV. In addition, $\beta$-catenin silencing attenuated TNF- $\alpha$-induced apoptosis in mesangial cells. The present results suggested that inhibition of Wnt/ $\beta$-catenin signaling by $\beta$-catenin silencing may be a promising strategy for therapeutic intervention in renal fibrotic disorders. However, various limitations of the present study should be considered. First, the in vivo experiments consisted of static observations and therefore did not fully describe the fibrosis process. Second, Wnt/ $/$-catenin inhibition was evaluated only in vitro in mesangial cells and not in the rat CKD model. Further studies are required to fully assess the potential of Wnt/ $\beta$-catenin signaling inhibition as a potential method of ameliorating renal fibrosis and CKD in vivo.

In conclusion, the present study demonstrated that $\beta$-catenin silencing by siRNA attenuated apoptosis and expression of fibrosis-associated markers in mesangial cells. Thus, Wnt/ $\beta$-catenin signaling inhibition may be a potential intervention strategy for renal fibrotic disorders in the future.

\section{Acknowledgements}

The present study was funded by the Science and Technology Fund of Guizhou Health and Family Planning Commission (grant no. gzwkj2014-2-133), National Natural Science Foundation of China (grant no. 81503398), China Postdoctoral Science Foundation (grant no. 2015M582372), Science and Technology Planning Project of Guangdong Province (grant no. 2016A020226032), Natural Science Foundation 
$\mathbf{A}$

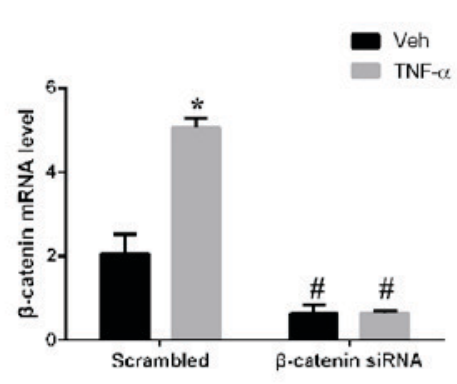

D

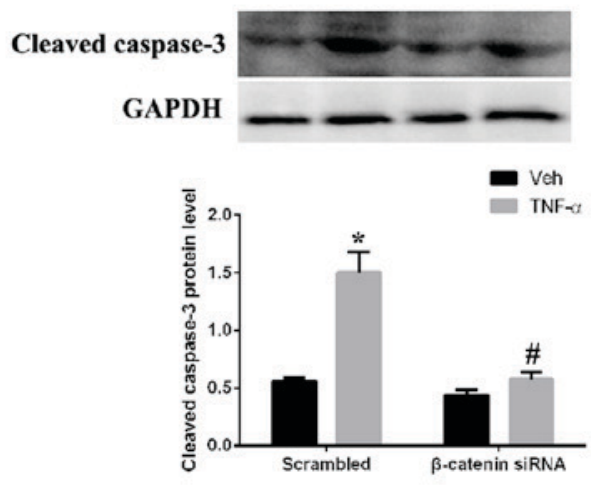

B

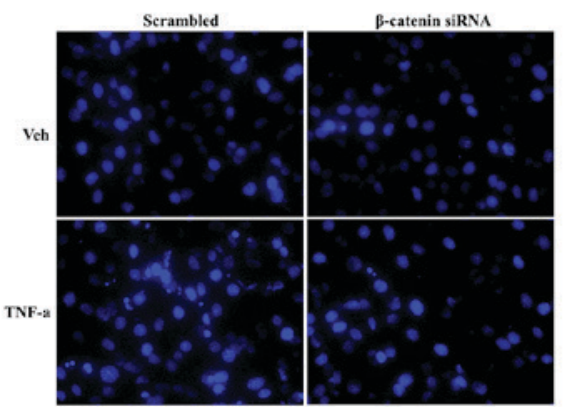

$\mathbf{E}$

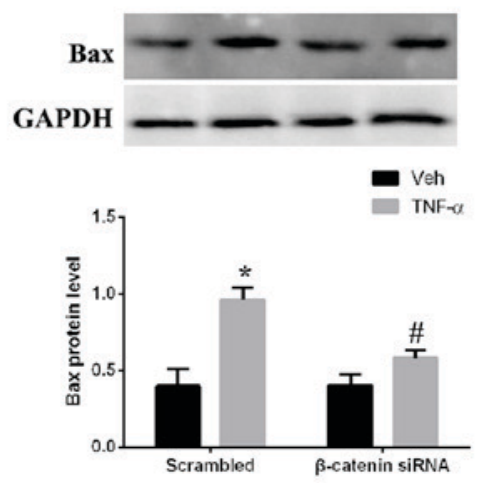

C

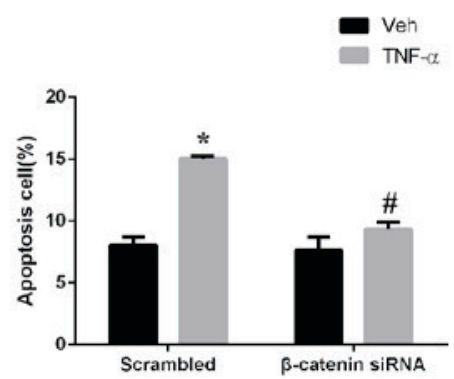

F

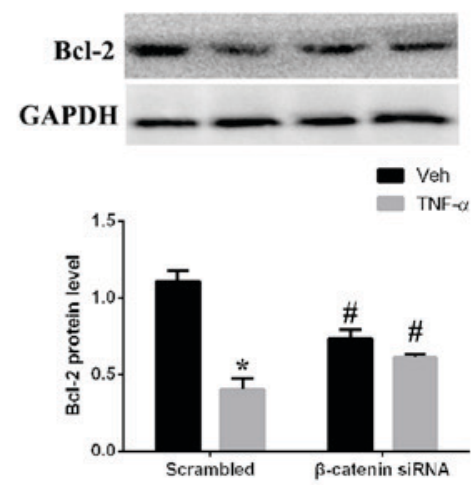

Figure 4. Silencing of $\beta$-catenin attenuates TNF- $\alpha$-induced apoptosis in mesangial cells. $\beta$-catenin expression was silenced in mesangial cells by transfection with $\beta$-catenin siRNA; control cells were transfected with a scrambled control. Cells were subsequently treated with vehicle or TNF- $\alpha$ for 24 h. (A) mRNA expression levels of $\beta$-catenin were determined by reverse transcription-quantitative polymerase chain reaction. (B) Representative images and (C) quantification of apoptosis following Hoechst 33258 staining. Western blot analysis of (D) cleaved caspase-3, (E) Bax and (F) Bcl-2 protein expression levels. Data are expressed as the mean \pm standard error. " $\mathrm{P}<0.05$ vs. vehicle control; ${ }^{*} \mathrm{P}<0.05$ vs. corresponding scrambled control. TNF- $\alpha$, tumor necrosis factor- $\alpha$; siRNA, small interfering RNA; Bax, B-cell lymphoma 2-associated X protein; Bcl-2, B-cell lymphoma 2; Veh, vehicle.

A

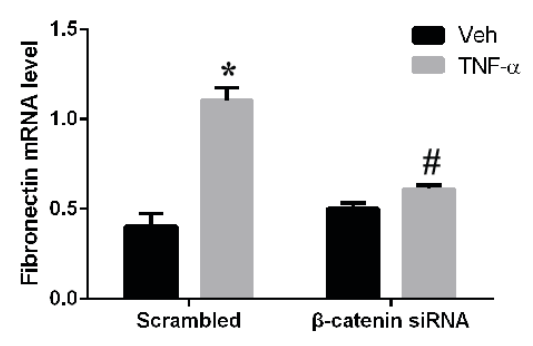

D
B

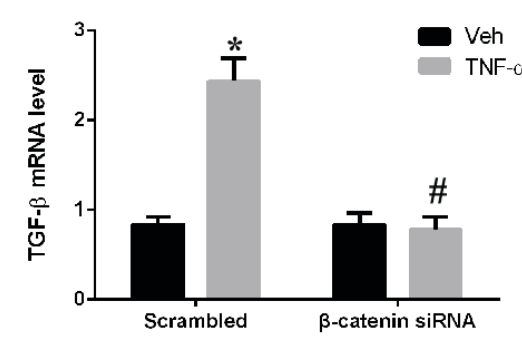

C

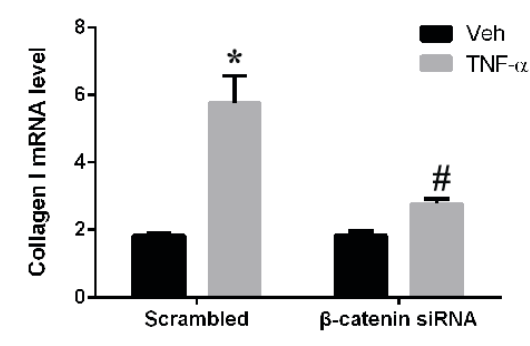

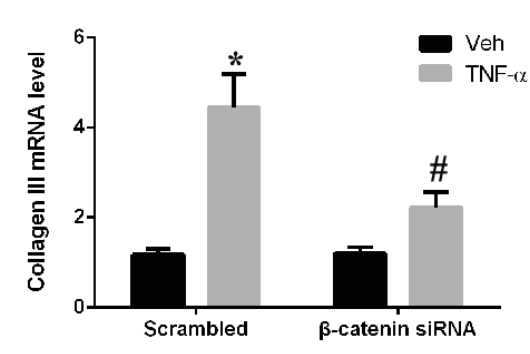

E

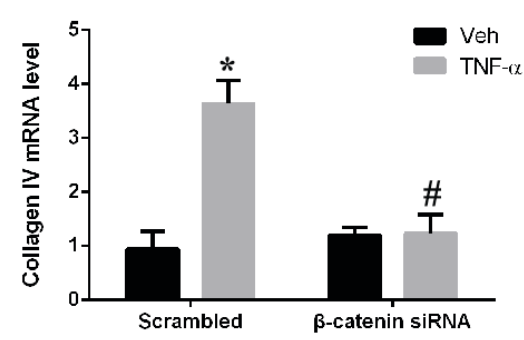

Figure 5. Silencing of $\beta$-catenin downregulates mRNA expression levels of fibrosis-associated genes. $\beta$-catenin expression was silenced in mesangial cells by transfection with $\beta$-catenin siRNA; control cells were transfected with a scrambled control. Cells were subsequently treated with vehicle or TNF- $\alpha$ for 24 h. mRNA expression levels of (A) fibronectin, (B) TGF- $\beta$, and (C) collagen I, (D) III and (E) IV were determined by reverse transcription-quantitative polymerase chain reaction. Data are expressed as the mean \pm standard error. ${ }^{*} \mathrm{P}<0.05$ vs. vehicle control; ${ }^{*} \mathrm{P}<0.05$ vs. corresponding scrambled control. siRNA, small interfering RNA; TNF- $\alpha$, tumor necrosis factor- $\alpha$; TGF- $\beta$, transforming growth factor- $\beta$; Veh, vehicle. 
of Guangxi Province (grant nos. 2015GXNSFBA139171 and 2016GXNSFAA380005), Shenzhen Science and Technology Project (grant nos. JCYJ20160428175036148 and JSGG20141017103353178), and Health and Family Planning Commission of Shenzhen Municipality (grant no. 201,605,013).

\section{References}

1. Kazancioğlu R: Risk factors for chronic kidney disease: An update. Kidney Int Suppl (2011) 3: 368-371, 2013.

2. Falke LL, Gholizadeh S, Goldschmeding R, Kok RJ and Nguyen TQ: Diverse origins of the myofibroblast-implications for kidney fibrosis. Nat Rev Nephrol 11: 233-244, 2015.

3. Guo Y, Xiao L, Sun L and Liu F: Wnt/beta-catenin signaling: A promising new target for fibrosis diseases. Physiol Res 61 : 337-346, 2012.

4. Sutariya B, Jhonsa D and Saraf M N: TGF- $\beta$ : The connecting link between nephropathy and fibrosis. Immunopharmacol Immunotoxicol 38: 39-49, 2016.

5. Wynn TA: Common and unique mechanisms regulate fibrosis in various fibroproliferative diseases. J Clin Invest 117: 524-529, 2007.

6. Kriz W, Kaissling B and Le Hir M: Epithelial-mesenchymal transition (EMT) in kidney fibrosis: Fact or fantasy? J Clin Invest 121: 468-474, 2011.

7. Eger A, Stockinger A, Park J, Langkopf E, Mikula M, Gotzmann J, Mikulits W, Beug H and Foisner R: Beta-catenin and TGFbeta signalling cooperate to maintain a mesenchymal phenotype after FosER-induced epithelial to mesenchymal transition. Oncogene 23: 2672-2680, 2004.

8. Sato M: Upregulation of the Wnt/beta-catenin pathway induced by transforming growth factor-beta in hypertrophic scars and keloids. Acta Derm Venereol 86: 300-307, 2006.

9. Cheng JH, She H, Han YP, Wang J, Xiong S, Asahina K and Tsukamoto H: Wnt antagonism inhibits hepatic stellate cell activation and liver fibrosis. Am J Physiol Gastrointest Liver Physiol 294: G39-G49, 2008.

10. Wei J, Melichian D, Komura K, Hinchcliff M,Lam AP, Lafyatis R, Gottardi CJ, MacDougald OA and Varga J: Canonical Wnt signaling induces skin fibrosis and subcutaneous lipoatrophy: A novel mouse model for scleroderma? Arthritis Rheum 63 : $1707-1717,2011$

11. Zhou T, He X, Cheng R, Zhang B, Zhang RR, Chen Y, Takahashi Y, Murray AR, Lee K, Gao G and Ma JX: Implication of dysregulation of the canonical wingless-type MMTV integration site (WNT) pathway in diabetic nephropathy. Diabetologia 55: 255-266, 2012

12. He W, Dai C,Li Y,Zeng G, Monga SP and Liu Y: Wnt/beta-catenin signaling promotes renal interstitial fibrosis. J Am Soc Nephrol 20: 765-776, 2009.

13. Hao S, He W, Li Y, Ding H, Hou Y, Nie J, Hou FF, Kahn M and Liu Y: Targeted inhibition of $\beta$-catenin/CBP signaling ameliorates renal interstitial fibrosis. J Am Soc Nephrol 22: 1642-1653, 2011.

14. Akhmetshina A, Palumbo K, Dees C, Bergmann C, Venalis P, Zerr P, Horn A, Kireva T, Beyer C, Zwerina J, et al: Activation of canonical Wnt signalling is required for TGF- $\beta$-mediated fibrosis. Nat Commun 3: 735, 2012.
15. Arend RC, Londoño-Joshi AI, Samant RS, Li Y, Conner M, Hidalgo B, Alvarez RD, Landen CN, Straughn JM and Buchsbaum DJ: Inhibition of Wnt/ $\beta$-catenin pathway by niclosamide: A therapeutic target for ovarian cancer. Gynecol Oncol 134: 112-120, 2014

16. Wang C, Zhu H, Sun Z, Xiang Z, Ge Y, Ni C, Luo Z, Qian W and Han X: Inhibition of Wnt/ $\beta$-catenin signaling promotes epithelial differentiation of mesenchymal stem cells and repairs bleomycin-induced lung injury. Am J Physiol Cell Physiol 307: C234-C244, 2014.

17. Tanjore H, Degryse AL, Crossno PF, Xu XC, McConaha ME, Jones BR, Polosukhin VV, Bryant AJ, Cheng DS, Newcomb DC, et al: $\beta$-Catenin in the alveolar epithelium protects from lung fibrosis after intratracheal bleomycin. Am J Respir Crit Care Med 187: 630-639, 2013

18. Wang DT, Huang RH, Cheng X, Zhang ZH, Yang YJ and Lin X: Tanshinone IIA attenuates renal fibrosis and inflammation via altering expression of TGF- $\beta /$ Smad and $\mathrm{NF}-\kappa \mathrm{B}$ signaling pathway in 5/6 nephrectomized rats. Int Immunopharmacol 26: 4-12, 2015.

19. Livak KJ and Schmittgen TD: Analysis of relative gene expression data using real-time quantitative PCR and the 2-(Delta Delta C(T)) method. Methods 25: 402-408, 2001.

20. Verma UN, Surabhi RM, Schmaltieg A, Becerra C and Gaynor RB: Small interfering RNAs directed against $\beta$-catenin inhibit the in vitro and in vivo growth of colon cancer cells. Clin Cancer Res 9: 1291-1300, 2003.

21. Schneider CA, Rasband WS and Eliceiri KW: NIH Image to ImageJ: 25 years of image analysis. Nat Methods 9: 671-675, 2012.

22. Tan RJ, Zhou D, Zhou L and Liu Y: Wnt/ $\beta$-catenin signaling and kidney fibrosis. Kidney Int Suppl (2011) 4: 84-90, 2014.

23. Zhou D, Tan RJ, Fu H and Liu Y: Wnt//-catenin signaling in kidney injury and repair: A double-edged sword. Lab Invest 96: 156-167, 2016.

24. Peng $J$ and Dong $Z$ : Role changes of $\beta$-catenin in kidney injury and repair. Kidney Int 82: 509-511, 2012.

25. Dai C, Stolz DB, Kiss LP, Monga SP, Holzman LB and Liu Y: Wnt/beta-catenin signaling promotes podocyte dysfunction and albuminuria. J Am Soc Nephrol 20: 1997-2008, 2009.

26. von Toerne C, Schmidt C, Adams J, Kiss E, Bedke J, Porubsky S, Gretz N, Lindenmeyer MT, Cohen CD, Gröne HJ and Nelson PJ: Wnt pathway regulation in chronic renal allograft damage. Am J Transplant 9: 2223-2239, 2009.

27. Xiao L, Zhou D, Tan RJ, Fu H, Zhou L, Hou FF and Liu Y: Sustained activation of $\mathrm{Wnt} / \beta$-Catenin signaling drives AKI to CKD progression. J Am Soc Nephrol 27: 1724-1740, 2016.

28. Lin CL, Cheng H, Tung CW, Huang WJ, Chang PJ, Yang JT and Wang JY: Simvastatin reverses high glucose-induced apoptosis of mesangial cells via modulation of Wnt Signaling pathway. Am J Nephrol 28: 290-297, 2008.

29. Haramis AP, Begthel H, van den Born M, van Es J, Jonkheer S, Offerhaus GJ and Clevers H: De novo crypt formation and juvenile polyposis on BMP inhibition in mouse intestine. Science 30 : 1684-1686, 2004.

30. Polakis P: The many ways of Wnt in cancer. CurrOpin Genet Dev 17: 45-51, 2007.

31. Zhou L, Li Y, Zhou D, Tan RJ and Liu Y: Loss of Klotho contributes to kidney injury by derepression of Wnt $/ \beta$-Catenin signaling. J Am Soc Nephrol 24: 771-785, 2013. 